\title{
Predictors of Recovery from Traumatic Brain Injury-Induced Prolonged Consciousness Disorder
}

\author{
Hiroaki Abe, ${ }^{1,2}$ Keigo Shimoji, ${ }^{3}$ Yoshihide Nagamine, ${ }^{4}$ \\ Satoru Fujiwara, ${ }^{5}$ and Shin-Ichi Izumi ${ }^{2,6}$ \\ ${ }^{1}$ Department of Rehabilitation Medicine, Kohnan Hospital, Tohoku Ryogo Center, Sendai, Japan \\ ${ }^{2}$ Department of Physical Medicine and Rehabilitation, Graduate School of Medicine, \\ Tohoku University, Sendai, Japan \\ ${ }^{3}$ Department of Diagnostic Radiology, Tokyo Metropolitan Geriatric Hospital, Tokyo, Japan \\ ${ }^{4}$ Department of Neurosurgery, Kohnan Hospital, Tohoku Ryogo Center, Sendai, Japan \\ ${ }^{5}$ Department of Neurosurgery, Kohnan Hospital, Sendai, Japan \\ ${ }^{6}$ Graduate School of Biomedical Engineering, Tohoku University, Sendai, Japan
}

Correspondence should be addressed to Hiroaki Abe; abehi0827@gmail.com

Received 28 October 2016; Revised 19 January 2017; Accepted 22 January 2017; Published 23 February 2017

Academic Editor: Cheng-Sheng Chen

Copyright (C) 2017 Hiroaki Abe et al. This is an open access article distributed under the Creative Commons Attribution License, which permits unrestricted use, distribution, and reproduction in any medium, provided the original work is properly cited.

\begin{abstract}
We investigated the clinical predictors of the degree of recovery in patients with prolonged disorders of consciousness (PDC) caused by traumatic brain injury. Fourteen patients with PDC underwent two diffusion tensor imaging (DTI) studies; the first and second scans were performed at $345.6 \pm 192.6$ and $689.1 \pm 272.2$ days after the injury, respectively. In addition to the temporal changes in each of these diffusion parameters, fractional anisotropy (FA), mean diffusivity, axial diffusivity (AD), and radial diffusivity were assessed over a 1-year period. Relationship of clinical and DTI parameters with recovery from PDC (RPDC) was evaluated using Spearman's rank-correlation and stepwise multiple linear regression analysis. The mean FA and number of voxels with FA values $>$ 0.4 (VsFA0.4) were significantly decreased at the second scan. A significant positive correlation was observed between the degree of RPDC and mean FA $(r=0.60)$ and VsFA0.4 $(r=0.68)$ as well as between the difference in VsFA0.4 $(r=0.63)$ and AD $(r=0.54)$ between the first and second scans. On multiple linear regression analysis, initial severity of PDC and the difference in AD remained significantly associated with the degree of RPDC. The microstructural white matter changes observed in this study indicate their potential relation with the degree of RPDC over the longer term.
\end{abstract}

\section{Introduction}

Diffuse axonal injury (DAI) is a common form of traumatic brain injury (TBI) sustained in motor vehicle collisions. The injury involves rotational forces and is characterized by extensive white matter damage [1]. The neuropathology of disorders of consciousness (coma, vegetative state, and minimally conscious state) has been extensively described at postmortem [2-4]. Diffuse disruption of subcortical white matter is the most common postmortem finding in victims of TBI associated with impaired consciousness [2]. DAI and thalamic damage were the most common postmortem structural abnormalities reported in a case series of 35 patients who remained in a vegetative state after TBI until the time of their death $[3,4]$. Thus, severe TBI commonly involves multiple diffuse lesions in both white and gray matter.

Diffusion tensor imaging (DTI) has been useful in describing the microstructure changes in the chronic stage of DAI [5]. Several studies have documented posttraumatic findings of axotomy and demyelination, months to years after injury. These include findings of increased water diffusion as measured by mean diffusivity (MD) and a reduction in the directionality of diffusion as measured by fractional anisotropy (FA) on DTI [5]. These findings, in conjunction with the apparent loss of subcortical white matter volume, suggest that acute edema may be a potential early marker 
of posttraumatic deterioration that ultimately impairs axonal integrity in the chronic state [5]. Few longitudinal studies have examined temporal evolution of white matter damage after DAI in humans [6-9]. However, the acute scans were collected, on average, within two months after injury; smaller time windows are necessary to obtain a more thorough understanding of the evolution of white matter damage. Further, several studies have documented correlation between decreased FA in several brain regions and unfavorable outcomes in patients with severe TBI $[8,10,11]$. A recent whole brain WM analysis of DTI parameters revealed an increase in axial diffusivity (AD) and radial diffusivity (RD) in the acute phase and a positive correlation of RD with severity of injury [5]. Longitudinal analysis showed reduction in FA and $\mathrm{AD}$, but not in RD [5]. This study [5] examined the evolution of white matter integrity from acute to chronic stages of DAI due to TBI. Data from this study suggest complicated mild-to-severe TAI results in significant edema that eventually resolves leaving behind a compromised white matter microstructure. The data [5] also suggest that white matter compromise after DAI is a process involving white matter demyelination as well as axonal damage that may be present not only in the early stage but also in the chronic stage [5]. However, the mechanism that explains this finding is still unclear. DTI still appears to be capable of detecting microstructural changes after DAI.

Patients with severe PDC, such as those in a vegetative state, typically have unfavorable outcomes although gradual, subtle, and minor clinical changes are on record. In the last 2 years, our institution provided inpatient care to patients with prolonged disorders of consciousness (PDC) due to TBI. The therapeutic modalities include standard nursing care, physical therapy, and occupational therapy, as well as other alternative treatment modalities such as music therapy, aroma massage, and exposure to natural environment (i.e., feeling the sunlight, blowing wind, and exposure to seasonal temperature changes). Indeed, a few patients have shown slight positive reactions during inpatient residency at our institution.

In this study, we sought to identify potential predictors of the degree of recovery from PDC over a period of 2 years, using DTI parameters in whole brain. We examined the longitudinal alterations in anatomical connections of white matter in these patients and explored potential association of microstructural imaging biomarkers in whole brain white and gray matter with clinical markers of potential clinical relevance.

\section{Methods}

2.1. Patients. We retrospectively recruited 14 patients at our institution that had chronic severe PDC resulting from traffic accident-related brain injury. All patients with PDC underwent $3 \mathrm{~T}$ MRI studies at admission and after completion of 1 year of treatment at our institution. Mean age $( \pm S D)$ of patients was $58.6 \pm 19.3$ years; the mean duration $( \pm$ SD) from admission to study recruitment was $339.8 \pm 191.7$ days. The clinical characteristics of patients are presented in Table 1 . The first DTI scan was performed at admission (345.6 \pm 192.6 days after the initial injury); the second scan was performed approximately 1 year after admission $(689.0 \pm 272.2$ days after the initial injury).

Furthermore, we prospectively recruited 8 healthy normal volunteers ( 2 males and 6 females) for comparison. The age range of the healthy participants was $32-60(45.5 \pm 9.1)$ years (Table 2).

\subsection{Standard Protocol Approvals, Registrations, and Patient} Consent. This study was conducted in compliance with the ethical principles for biomedical research on human subjects enshrined in the Declaration of Helsinki and informed consent regulations. Approval from the institutional ethics committee was obtained prior to the initiation of the study.

2.3. PDC Assessment. PDC was assessed using the Kohnan score, which measures the severity of consciousness disorder from a severe, persistent vegetative state (Appendix [12]). The Kohnan score was developed at our institution to resolve this issue. The unidimensionality and higher intra- and interrater reliability of this score have previously been reported [12]. The score is based on seven parameters, each of which is subdivided into 5 grades: extreme (10 points), severe ( 9 points), moderate (7 or 8 points), mild (5 points), and slight ( 0 points). We additionally assessed general functional recovery using the Extended Glasgow Outcome Scale (GOSE) (range 1-8; higher scores indicate superior functional outcomes). These assessments were performed at admission and at 2 years after admission.

2.4. Image Acquisition. MRI was performed using a $3.0 \mathrm{~T}$ Signa Excite HD scanner (General Electric, Milwaukee, WI, USA). The parameters for DTI were as follows: echo time, $59 \mathrm{~ms}$; repetition time, $9,000 \mathrm{~ms}$; flip angle, $90^{\circ}$; slice thickness, $3 \mathrm{~mm}$ with no gap; field of view, $28.8 \times 28.8 \mathrm{~cm}$; acquisition matrix, $96 \times 96$; image matrix, $256 \times 256$ with a voxel size of $1.125 \times 1.125 \times 3.0 \mathrm{~mm}$; number of excitations, 1 ; and band width, $250 \mathrm{kHz}$. Images were obtained using 15directional diffusion encoding ( $b$ value, $1,000 \mathrm{~s} / \mathrm{mm}^{2}$ in each direction) and one set of images with $b=0 \mathrm{~s} / \mathrm{mm}^{2}$.

A total of 46 axial sections covering the entire cerebrum were obtained. The most inferior DTI slices were positioned at the medulla oblongata during acquisition.

2.5. Image Preprocessing. All DTI data were processed using the FSL software package (the Oxford Centre for Functional Magnetic Resonance Imaging of the Brain Software Library [13]). DTI images were skull-stripped using the Brain Extraction Tool (BET) in the FSL. The images were corrected using the Eddy Correct program of the FSL package to adjust for the effects of head movement and eddy currents. FA, MD, $\mathrm{AD}$, and $\mathrm{RD}$ maps were generated using dtifit program in the FSL. Lastly, we calculated the mean FA, MD, AD, and RD and counted the number of voxels in which FA values were $>0.4$ in the whole brain (VsFA0.4) using fslstats program implemented in the FSL. We assumed that using whole brain method affected not only white matter changes but also gray 


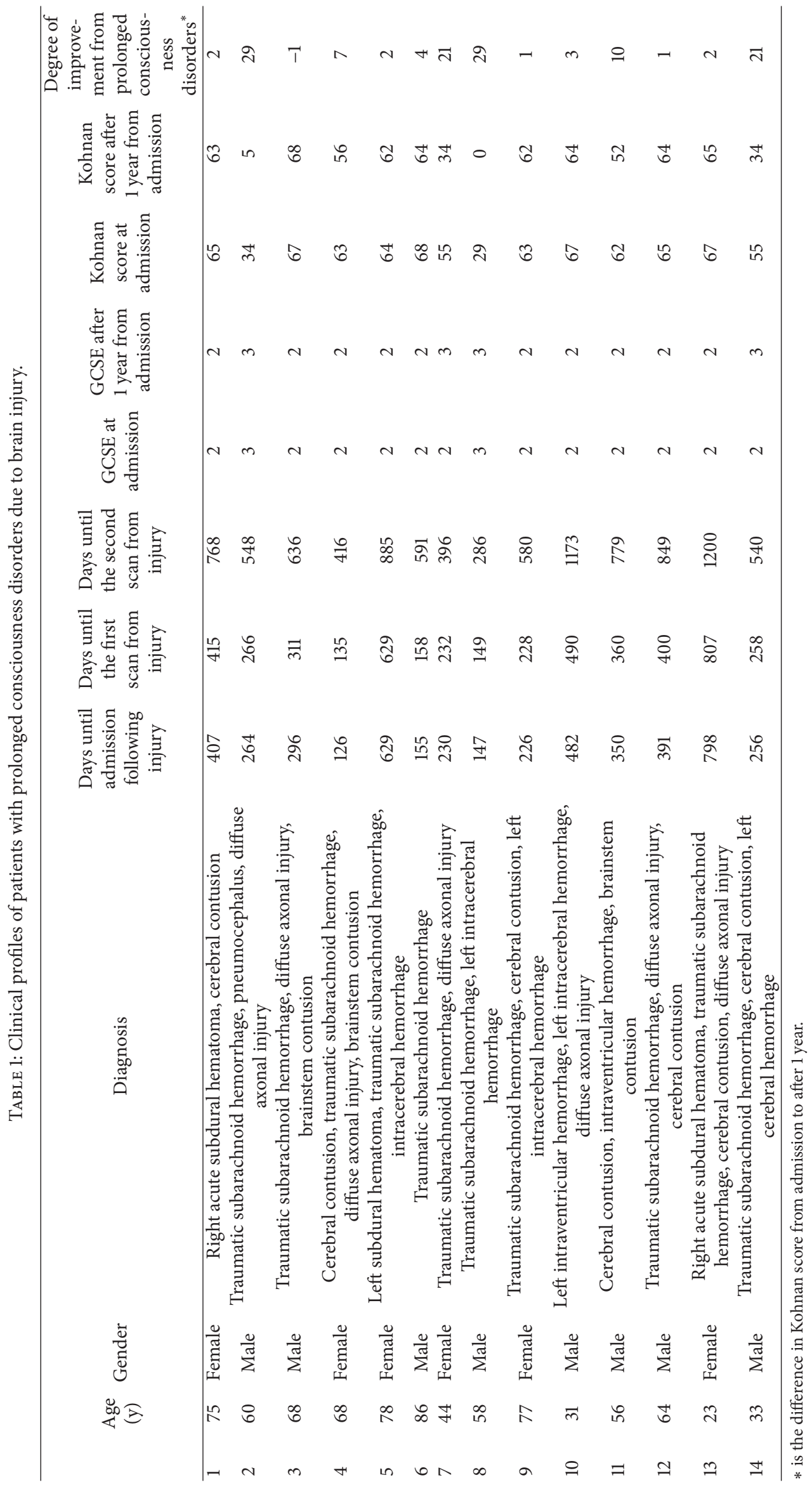




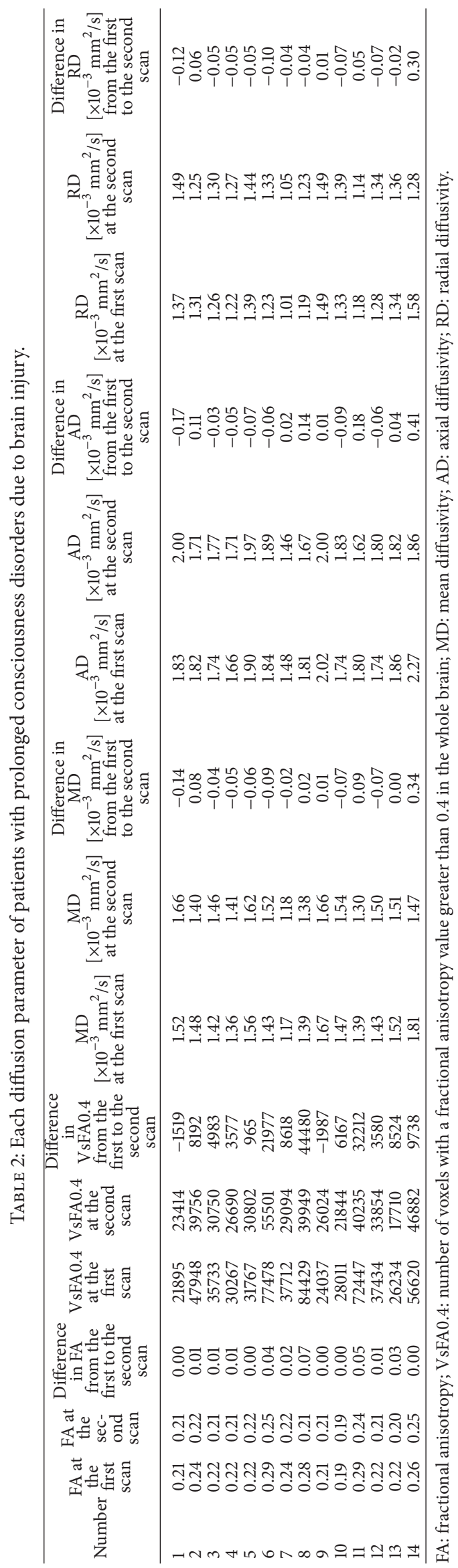


matter and ventricles. To eliminate these effects, we chose the method of VsFA0.4 that selects only the voxel with high FA values.

2.6. Statistical Analysis. Statistical analyses were performed using the Statistical Package for the Social Sciences (SPSS) 24.0 for Mac (IBM SPSS, Chicago, IL, USA). Comparisons over time for group Kohnan scores, GOSE, FA, VsFA0.4, $\mathrm{MD}, \mathrm{AD}$, and $\mathrm{RD}$ were conducted using the paired $t$-test or Wilcoxon rank-sum test according to the results of the Shapiro-Wilk test. The Spearman rank-correlation coefficient was used to evaluate the relationships between age, time of admission from injury, degree of recovery from PDC (as determined by change in the Kohnan score during the 2-year period after admission), and the diffusion tensor parameters. These diffusion tensor parameters used for comparisons were FA, VsFA0.4 MD, $\mathrm{AD}$, and $\mathrm{RD}$ in the first scan and the differences in the same parameters between the first and the second scan. All univariate potential factors with a $P$ value $<0.1$ were entered into the multivariate linear regression model. In addition, variance inflation factors were computed to examine the possible collinearity problem among the predictors. Stepwise multiple regression analyses were performed to identify the variables associated with the degree of recovery from PDC. The alpha level was set to 0.05 for all statistical tests and adjusted for multiple tests (e.g., $n=5, P>0.01$, comparison of the mean $\mathrm{FA}, \mathrm{MD}, \mathrm{AD}$, and RD) with Bonferroni correction.

\section{Results}

In this study, 12 patients had a GOSE score of 2 (vegetative state), and 2 patients had GOSE score of 3 (severe disorder) at admission. However, Kohnan scores at admission showed more variability (range: $29-68$ ). These ranged from minimum consciousness disorder $(<39)$ to complete vegetative state $(>65)$. After 2 years, there was no significant change in GOSE scores $(P=0.16$, Wilcoxon rank-sum test): 10 patients had a GOSE score of 2, while 4 patients had a GOSE score of 3 . In contrast, a subset of PDC patients showed a significant recovery based on the Kohnan score $(58.9 \pm 12.3$ versus $48.7 \pm$ 22.7, $P<0.01$; Wilcoxon rank-sum test) (Table 3 ). The mean difference in Kohnan scores at admission and at completion of 2 years was $10.14 \pm 11.10$.

In patients with $\mathrm{PDC}, \mathrm{FA}, \mathrm{VsFA0.4}, \mathrm{MD}, \mathrm{AD}$, and $\mathrm{RD}$ values in the first scan were $0.24 \pm 0.03,43715.14 \pm 20913.54$, $1.47 \pm 0.15\left[\times 10^{-3} \mathrm{~mm}^{2} / \mathrm{s}\right], 1.82 \pm 0.18\left[\times 10^{-3} \mathrm{~mm}^{2} / \mathrm{s}\right]$, and $1.30 \pm 0.14\left[\times 10^{-3} \mathrm{~mm}^{2} / \mathrm{s}\right]$, respectively, whereas those in the second scan were $0.22 \pm 0.02,33036.07 \pm 10413.59,1.47 \pm$ $0.01\left[\times 10^{-3} \mathrm{~mm}^{2} / \mathrm{s}\right], 1.79 \pm 0.15\left[\times 10^{-3} \mathrm{~mm}^{2} / \mathrm{s}\right]$, and $1.31 \pm$ $0.13\left[\times 10^{-3} \mathrm{~mm}^{2} / \mathrm{s}\right]$, respectively (Table 3$)$. Patients with PDC showed significant changes in FA $(P=0.006$, paired $t$-test $)$ and VsFA0.4 ( $P=0.003$, Wilcoxon rank-sum test $)$ over time. However, there were no significant differences in MD ( $P=0.993$, paired $t$-test $), \mathrm{AD}(P=0.506$, paired $t$-test $)$, and $\mathrm{RD}(P=0.650$, paired $t$-test $)$ values between the first and second scans (Table 4 ). In contrast, any significant change of DTI parameters was not shown in the 8 normal healthy participants (Table 5).
No significant correlations were observed between the degree of recovery from PDC and age. Kohnan score was strongly associated $(r=-0.76, P=0.002)$ with the degree of recovery from PDC. Among the DTI parameters, significant correlations were observed between the degree of recovery from PDC and FA $(r=0.60, P<0.05)$ and VsFA0.4 at the first scan $(r=0.68, P=0.008)$; however, no significant correlation was observed between the $\mathrm{MD}, \mathrm{AD}$, and $\mathrm{LD}$ at the first scan (Table 6). In the longitudinal DTI parameters, no significant correlations were observed between the degree of recovery from PDC and difference in FA, MD, and RD over time (Table 6). However, a significant positive correlation was observed between the degree of recovery from PDC and timedependent changes in AD $(r=0.544, P<0.05)$ and VsFA0.4 $(r=0.624, P<0.05)$ between the first and second scans (Table 6).

Variables showing a significant association $(P$ value $<0.1)$ were included in the Spearman correlation analysis. Thus, day of institution admission from injury, Kohnan score at the first assessment, FA at the first scan, VsFA0.4 at the first scan, difference in VsFA0.4, and MD and AD from the first to the second scan were included in the multivariate regression analysis. Stepwise multiple regression analysis revealed that the Kohnan score at the first scan (standardized $\beta=-0.723$, 95\% CI: 32.7-63.0, $P<0.0001)$ and difference in AD over time (standardized $\beta=0.337,95 \% \mathrm{CI}$ : 4610.7-46541.3, $P<$ 0.05 ) accurately predicted the degree of recovery from PDC [adjusted $R^{2}=0.841$ ]

\section{Discussion}

We explored potential clinical predictors of the degree of recovery from PDC after 2 years of admission, with a particular focus on the DTI parameters. Our results showed significantly decreased FA values and number of voxels in which FA values were $>0.4$ (VsFA0.4) in whole brain, but $\mathrm{MD}, \mathrm{AD}$, and $\mathrm{RD}$ showed no significant change. In contrast, healthy participants did not exhibit such changes. On correlation analysis, Kohnan score and FA and VsFA0.4, the difference of VsFA0.4, and AD were significantly correlated in monovariable analysis. Multiple regression analysis revealed that each Kohan score in the first assessment and the difference in $\mathrm{AD}$ over time have statistically significant contribution to the degree of recovery from PDC. Currently, to the best of our knowledge, there are no known predictors of long-term positive clinical response in patients with PDC. Several clinicians were forced to provide long-term care without established specific clinical goals for patients with PDC. Therefore, it is critical to develop predictors of long-term changes elicited through long-term therapeutic intervention in these patients. Our results demonstrate that microstructural white matter changes occurred in patients with PDC, which suggests that the assessment of white matter changes may help identify valid long-term outcome predictors in these patients.

DTI is based upon the diffusivity of water molecules, which varies in different tissues [14]. In white matter, it is more limited in the directions of diffusion. In healthy tracts, the anisotropy (limited directionality of diffusion) is higher 


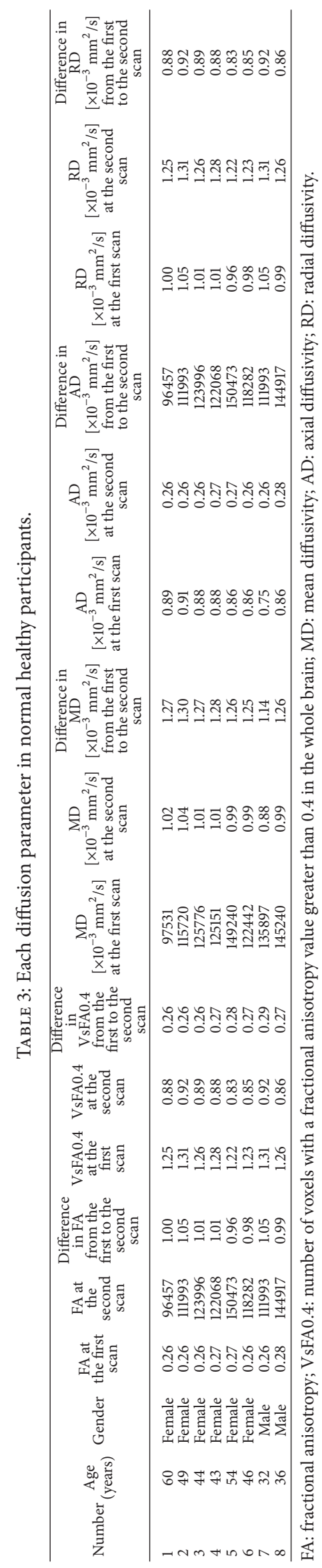


TABLE 4: Comparison of diffusion tensor parameters and consciousness disorders.

\begin{tabular}{|c|c|c|c|c|c|}
\hline \multirow[b]{2}{*}{ Diffusion parameters } & \multicolumn{2}{|c|}{ First scan } & \multicolumn{2}{|c|}{ Second scan } & \multirow[b]{2}{*}{$P$ values } \\
\hline & Mean & $\begin{array}{l}\text { Standard } \\
\text { deviation }\end{array}$ & Mean & $\begin{array}{l}\text { Standard } \\
\text { deviation }\end{array}$ & \\
\hline Kohnan score $^{*}$ & 58.86 & 12.30 & 48.71 & 22.69 & 0.001 \\
\hline GSCE* $^{*}$ & 2.14 & 0.36 & 2.29 & 0.47 & 0.157 \\
\hline Fractional anisotropy & 0.24 & 0.03 & 0.22 & 0.02 & 0.006 \\
\hline $\begin{array}{l}\text { Number of voxels with a fractional anisotropy value greater } \\
\text { than } 0.4 \text { in the whole brain* }\end{array}$ & 43715.14 & 20913.55 & 33036.07 & 10413.59 & 0.003 \\
\hline Mean diffusivity (MD) $\left[\times 10^{-3} \mathrm{~mm}^{2} / \mathrm{s}\right]$ & 1.47 & 0.15 & 1.47 & 0.01 & 0.993 \\
\hline Axial diffusivity $(\mathrm{AD})\left[\times 10^{-3} \mathrm{~mm}^{2} / \mathrm{s}\right]$ & 1.82 & 0.18 & 1.79 & 0.15 & 0.506 \\
\hline Radial diffusivity (RD) $\left[\times 10^{-3} \mathrm{~mm}^{2} / \mathrm{s}\right]$ & 1.30 & 0.14 & 1.31 & 0.13 & 0.65 \\
\hline
\end{tabular}

${ }^{*}$ Wilcoxon rank test.

TABLE 5: Comparison of diffusion tensor parameters in normal healthy participants.

\begin{tabular}{|c|c|c|c|c|c|}
\hline \multirow[b]{2}{*}{ Diffusion parameters } & \multicolumn{2}{|c|}{ First scan } & \multicolumn{2}{|c|}{ Second scan } & \multirow[b]{2}{*}{$P$ values } \\
\hline & Mean & $\begin{array}{l}\text { Standard } \\
\text { deviation }\end{array}$ & Mean & $\begin{array}{l}\text { Standard } \\
\text { deviation }\end{array}$ & \\
\hline Fractional anisotropy & 0.26 & 0.07 & 0.27 & 0.01 & 0.13 \\
\hline $\begin{array}{l}\text { Number of voxels with a fractional anisotropy value greater than } 0.4 \\
\text { in the whole brain }\end{array}$ & 122522.38 & 17745.24 & 127124.63 & 16594.75 & 0.15 \\
\hline Mean diffusivity (MD) $\left[\times 10^{-3} \mathrm{~mm}^{2} / \mathrm{s}\right]^{*}$ & 1.01 & 0.03 & 0.99 & 0.05 & 0.40 \\
\hline Axial diffusivity (AD) $\left[\times 10^{-3} \mathrm{~mm}^{2} / \mathrm{s}\right]^{*}$ & 1.26 & 0.03 & 1.25 & 0.05 & 0.23 \\
\hline Radial diffusivity (RD) $\left[\times 10^{-3} \mathrm{~mm}^{2} / \mathrm{s}\right]^{*}$ & 0.88 & 0.03 & 0.09 & 0.05 & 0.57 \\
\hline
\end{tabular}

${ }^{*}$ Wilcoxon rank test.

TABLE 6: Correlation between the degree of recovery from prolonged consciousness disorder and each value.

\begin{tabular}{lc}
\hline Variable & $\begin{array}{c}\text { Degree of recovery from PCD } \\
r\end{array}$ \\
& $\begin{array}{c}\text { (Spearman correlation coefficient) } \\
\text { At the first scan or assessment }\end{array}$ \\
Age & -0.24 \\
Day of institution admission from injury & -0.48 \\
Kohnan score & -0.76 \\
Fractional anisotropy & 0.60 \\
Numbers of voxels with a fractional anisotropy value greater than 0.4 & 0.68 \\
Mean diffusivity & -0.22 \\
Axial diffusivity & -0.01 \\
Radial diffusivity & -0.34 \\
The difference between the first and the second scan & 0.086 \\
Fractional anisotropy & 0.002 \\
Numbers of voxels with a fractional anisotropy value greater than 0.4 & 0.38 \\
Mean diffusivity & 0.023 \\
Axial diffusivity & 0.42 \\
Radial diffusivity & 0.459 \\
\hline
\end{tabular}

than that in the gray matter. This difference allows for the calculation of fractional anisotropy (FA) values for tissue and the generation of white matter fiber maps. FA ranges from 0 to 1 , where 0 represents isotropic diffusion (or lack of directional organization) and 1 represents anisotropic diffusion (or organized tissue such as white matter tracts) [15]. Studies have demonstrated the potential utility of DTI for providing quantitative assessments of microstructural damage in TBI, in which DAI is common [1-11,14-16]. Although the specifics are still not well understood, FA is believed to be influenced by many factors, including the degree of myelination and axonal density and/or integrity [9, 17-19]. 


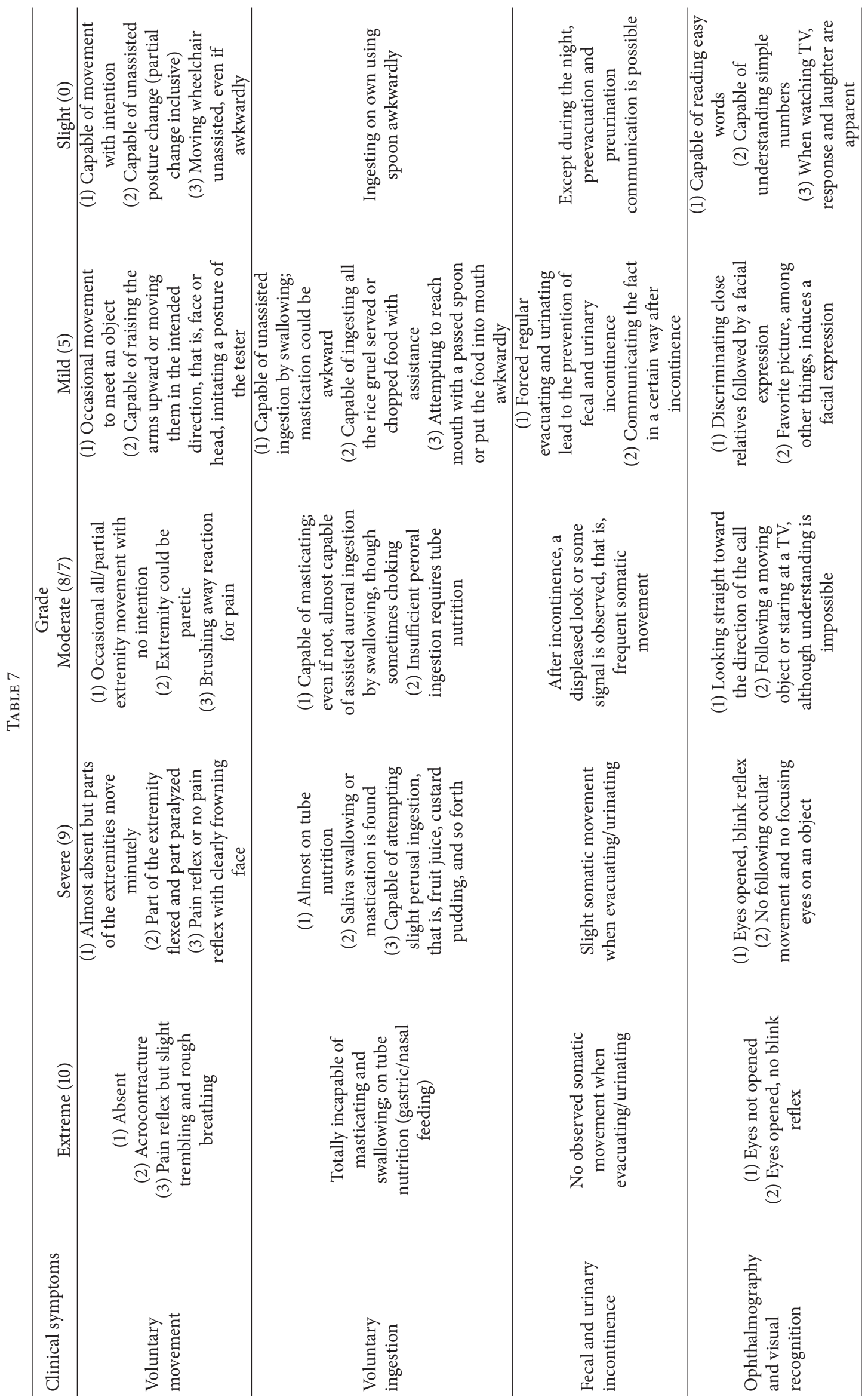




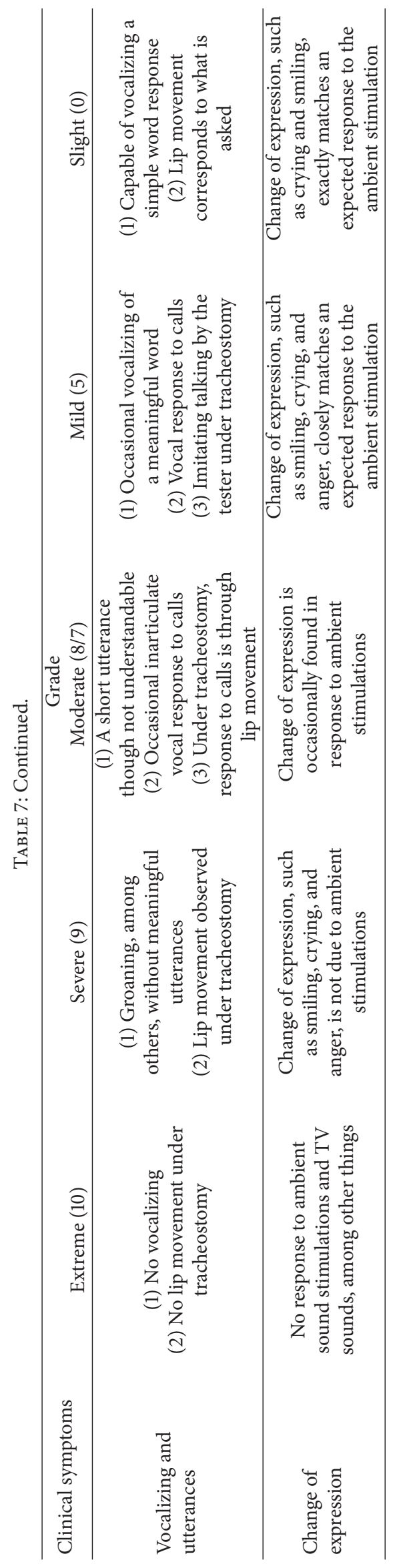


FA has been shown to decrease in patients with mild and moderate/severe brain injury [1, 4, 5, 11, 14-16, 19-28]. Perez et al. [5] reported significantly lower FA in the chronic phase of TBI as compared to that in healthy controls; in contrast, $\mathrm{AD}, \mathrm{MD}$, and $\mathrm{RD}$ in chronic patients were significantly higher than in healthy controls. Additionally, chronic FA showed a positive correlation with processing speed [28]. In this study, patients with chronic PDC had significantly lower FA and VsFA0.4 at 2 years as compared to those at admission. Our first scan data were collected $345.6 \pm 192.6$ days after injury, which corresponds to the chronic stage of white matter damage. Thus, our results suggest occurrence of microstructural change over the longer term. On univariate correlation analysis, FA and VsFA0.4 at the first scan significantly correlated with the degree of recovery of $\mathrm{PDC}$, which is consistent with previous studies [5-8, 10, 11, 14, 20-29] where patients with higher FA showed good recovery. Temporal change in VsFA0.4 and AD significantly correlated with the degree of recovery from PDC. This probably indicates that minor microstructural changes correlate with the degree of recovery from PDC. Several previous studies $[6,8,10,11$, 20, 25-29] have reported a positive association of FA with higher cognitive function and outcomes. However, in this study, higher difference of $\mathrm{VsFA0.4}, \mathrm{MD}$, and $\mathrm{AD}$ positively correlated with the degree of recovery from PDC. In other words, a time-dependent decrease in VsFA0.4, MD, and $\mathrm{AD}$ may be related to a better outcome in PDC. In this respect, our results are not inconsistent with those of several previous reports $[6,8,10,11,20,25-29]$, which indicated that a time-dependent decrease in FA was associated with poor outcomes as assessed by cognitive function and GOSE. These contradictory findings may reflect the difference in PDC severity between studies; most of our patients had GOSE scores of $\leq 3$ and showed subtle and minor clinical changes. Although significant recoveries were observed based on Kohnan scores, the changes were not significant when based on GOSE scores, which suggests that improvement in our patients was lower than that reported in previous studies $[6,8,10,11,20,25-29]$. Our subjects generally had more severe outcomes; all our patients had GOSE scores $\leq$ 3 , which indicates more severe disability than that among patients in the previous studies $[6,8,10,11,20,25-29]$. Thus, interstudy differences in results may reflect DAI severity. Differences with respect to time elapsed since injury may also have contributed to the divergent findings; we included patients with PDC in the extended chronic phase.

In patients with PDC, the degree of recovery was very small, as shown by the lack of change in GOSE scores. In our participants, induction of a severe inactive state due to brain injury might lead to secondary neurodegeneration that might be detected by DTI. However, the underlying mechanism behind longitudinal alterations in white matter remains unclear. In addition, this study had some limitations. The number of subjects was small, and they had various pathological states, such as contusion, subarachnoid hemorrhage, and intracranial hemorrhage. Thus, further longitudinal studies are warranted that combine DTI with volumetric measurements and other detailed analyses, such as functional connectivity analysis. The potential of DTI use as a prognostic tool needs further investigation in studies with a larger number of subjects.

In conclusion, on stepwise multiple linear regression analysis, Kohnan score and the difference in AD showed a significant association with the degree of recovery from PDC. In other words, we demonstrate evidence of temporal microstructural white matter changes in patients with PDC; DTI parameters are useful indices for assessment of white matter alterations in these patients. As a noninvasive modality, DTI provides in vivo quantitative pathophysiological information. Tracking white matter microstructural changes over time has the potential to measure neuroplasticity and repair after TBI and may eventually be utilized to monitor therapeutic responses. Further research is required to investigate the leads identified in this study.

\section{Appendix}

See Table 7 .

\section{Competing Interests}

The authors declare that they have no competing interests.

\section{Acknowledgments}

This study was supported by a medical research grant on traffic accidents from the General Insurance Association of Japan. This work was also supported by JSPS KAKENHI Grant no. JP16H06280, Grant-in-Aid for Scientific Research on Innovative Areas-Resource and Technical Support Platforms for Promoting Research "Advanced Bioimaging Support." The authors would like to thank the patients and normal healthy volunteers who participated in this study. The authors would also like to thank the imaging staff, particularly Tomohiro Chiba, for their skilled MRI acquisition. They also thank Enago for the English language review.

\section{References}

[1] M. A. Warner, C. M. De La Plata, J. Spence et al., "Assessing spatial relationships between axonal integrity, regional brain volumes, and neuropsychological outcomes after traumatic axonal injury," Journal of Neurotrauma, vol. 27, no. 12, pp. 21212130, 2010.

[2] H. C. Kinney and M. A. Samuels, "Neuropathology of the persistent vegetative state. A review," Journal of Neuropathology and Experimental Neurology, vol. 53, no. 6, pp. 548-558, 1994.

[3] J. H. Adams, B. Jennett, D. R. McLellan, L. S. Murray, and D. I. Graham, "The neuropathology of the vegetative state after head injury," Journal of Clinical Pathology, vol. 52, no. 11, pp. 804-806, 1999.

[4] D. Fernández-Espejo, T. Bekinschtein, M. M. Monti et al., "Diffusion weighted imaging distinguishes the vegetative state from the minimally conscious state," NeuroImage, vol. 54, no. 1, pp. 103-112, 2011.

[5] A. M. Perez, J. Adler, N. Kulkarni et al., "Longitudinal white matter changes after traumatic axonal injury," Journal of Neurotrauma, vol. 31, no. 17, pp. 1478-1485, 2014. 
[6] E. A. Wilde, S. R. McCauley, A. Barnes et al., "Serial measurement of memory and diffusion tensor imaging changes within the first week following uncomplicated mild traumatic brain injury," Brain Imaging and Behavior, vol. 6, no. 2, pp. 319-328, 2012.

[7] K. D. M. Farbota, A. Sodhi, B. B. Bendlin et al., "Longitudinal volumetric changes following traumatic brain injury: A Tensorbased Morphometry Study," Journal of the International Neuropsychological Society, vol. 18, no. 6, pp. 1006-1018, 2012.

[8] A. Sidaros, A. W. Engberg, K. Sidaros et al., "Diffusion tensor imaging during recovery from severe traumatic brain injury and relation to clinical outcome: a longitudinal study," Brain, vol. 131, no. 2, pp. 559-572, 2008.

[9] K. Arfanakis, V. M. Haughton, J. D. Carew, B. P. Rogers, R. J. Dempsey, and M. E. Meyerand, "Diffusion tensor MR imaging in diffuse axonal injury," American Journal of Neuroradiology, vol. 23, no. 5, pp. 794-802, 2002.

[10] K. Sugiyama, T. Kondo, Y. Oouchida et al., "Clinical utility of diffusion tensor imaging for evaluating patients with diffuse axonal injury and cognitive disorders in the chronic stage," Journal of Neurotrauma, vol. 26, no. 11, pp. 1879-1890, 2009.

[11] R. Kumar, M. Husain, R. K. Gupta et al., "Serial changes in the white matter diffusion tensor imaging metrics in moderate traumatic brain injury and correlation with neuro-cognitive function," Journal of Neurotrauma, vol. 26, no. 4, pp. 481-495, 2009.

[12] S. Fujiwara, N. Nakasato, Y. Nagamine et al., "Reliability and factorial structure of a rating scale for persistent vegetative state," Brain and Nerve, vol. 49, no. 12, pp. 1139-1145, 1997.

[13] S. M. Smith, "Fast robust automated brain extraction," Human Brain Mapping, vol. 17, no. 3, pp. 143-155, 2002.

[14] M. F. Kraus, T. Susmaras, B. P. Caughlin, C. J. Walker, J. A. Sweeney, and D. M. Little, "White matter integrity and cognition in chronic traumatic brain injury: a diffusion tensor imaging study," Brain, vol. 130, no. 10, pp. 2508-2519, 2007.

[15] D. Le Bihan, J.-F. Mangin, C. Poupon et al., "Diffusion tensor imaging: concepts and applications," Journal of Magnetic Resonance Imaging, vol. 13, no. 4, pp. 534-546, 2001.

[16] T. A. G. M. Huisman, L. H. Schwamm, P. W. Schaefer et al., "Diffusion tensor imaging as potential biomarker of white matter injury in diffuse axonal injury," AJNR American Journal of Neuroradiology, vol. 25, no. 3, pp. 370-376, 2004.

[17] S.-K. Song, S.-W. Sun, W.-K. Ju, S.-J. Lin, A. H. Cross, and A. H. Neufeld, "Diffusion tensor imaging detects and differentiates axon and myelin degeneration in mouse optic nerve after retinal ischemia," NeuroImage, vol. 20, no. 3, pp. 1714-1722, 2003.

[18] S.-K. Song, S.-W. Sun, M. J. Ramsbottom, C. Chang, J. Russell, and A. H. Cross, "Dysmyelination revealed through MRI as increased radial (but unchanged axial) diffusion of water," NeuroImage, vol. 17, no. 3, pp. 1429-1436, 2002.

[19] L. A. Harsan, P. Poulet, B. Guignard et al., "Brain dysmyelination and recovery assessment by noninvasive in vivo diffusion tensor magnetic resonance imaging," Journal of Neuroscience Research, vol. 83, no. 3, pp. 392-402, 2006.

[20] V. Veeramuthu, V. Narayanan, T. L. Kuo et al., "Diffusion tensor imaging parameters in mild traumatic brain injury and its correlation with early neuropsychological impairment: A Longitudinal Study," Journal of Neurotrauma, vol. 32, no. 19, pp. 1497-1509, 2015.

[21] J. M. Meythaler, J. D. Peduzzi, E. Eleftheriou, and T. A. Novack, "Current concepts: diffuse axonal injury-associated traumatic brain injury," Archives of Physical Medicine and Rehabilitation, vol. 82, no. 10, pp. 1461-1471, 2001.

[22] J. Ljungqvist, D. Nilsson, M. Ljungberg et al., "Longitudinal study of the diffusion tensor imaging properties of the corpus callosum in acute and chronic diffuse axonal injury," Brain Injury, vol. 25, no. 4, pp. 370-378, 2011.

[23] J. Y. Wang, K. Bakhadirov, H. Abdi et al., "Longitudinal changes of structural connectivity in traumatic axonal injury," Neurology, vol. 77, no. 9, pp. 818-826, 2011.

[24] J. Y. Wang, K. Bakhadirov, M. D. Devous Sr. et al., "Diffusion tensor tractography of traumatic diffuse axonal injury," Archives of Neurology, vol. 65, no. 5, pp. 619-626, 2008.

[25] G. Greenberg, D. J. Mikulis, K. Ng, D. DeSouza, and R. E. Green, "Use of diffusion tensor imaging to examine subacute white matter injury progression in moderate to severe traumatic brain injury," Archives of Physical Medicine and Rehabilitation, vol. 89, no. 12, pp. S45-S50, 2008.

[26] V. Perlbarg, L. Puybasset, E. Tollard, S. Lehéricy, H. Benali, and D. Galanaud, "Relation between brain lesion location and clinical outcome in patients with severe traumatic brain injury: a diffusion tensor imaging study using voxel-based approaches," Human Brain Mapping, vol. 30, no. 12, pp. 3924-3933, 2009.

[27] M. B. Hulkower, D. B. Poliak, S. B. Rosenbaum, M. E. Zimmerman, and M. L. Lipton, "A decade of DTI in traumatic brain injury: 10 years and 100 articles later," American Journal of Neuroradiology, vol. 34, no. 11, pp. 2064-2074, 2013.

[28] K. D. Farbota, B. B. Bendlin, A. L. Alexander, H. A. Rowley, R. J. Dempsey, and S. C. Johnson, "Longitudinal diffusion tensor imaging and neuropsychological correlates in traumatic brain injury patients," Frontiers in Human Neuroscience, vol. 6, article 160, 2012.

[29] P. M. Arenth, K. C. Russell, J. M. Scanlon, L. J. Kessler, and J. H. Ricker, "Corpus callosum integrity and neuropsychological performance after traumatic brain injury: a diffusion tensor imaging study," Journal of Head Trauma Rehabilitation, vol. 29, no. 2, pp. E1-E10, 2014. 

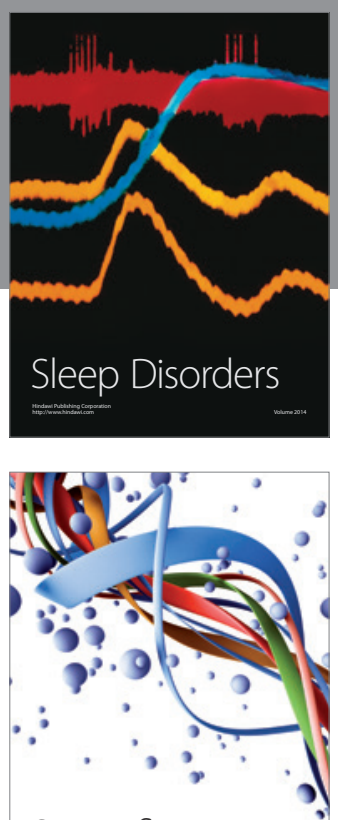

Scientifica
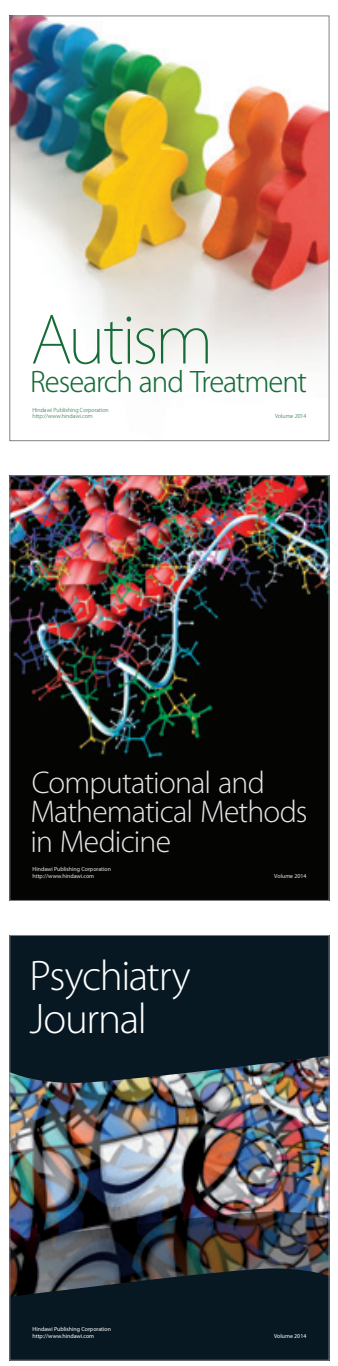
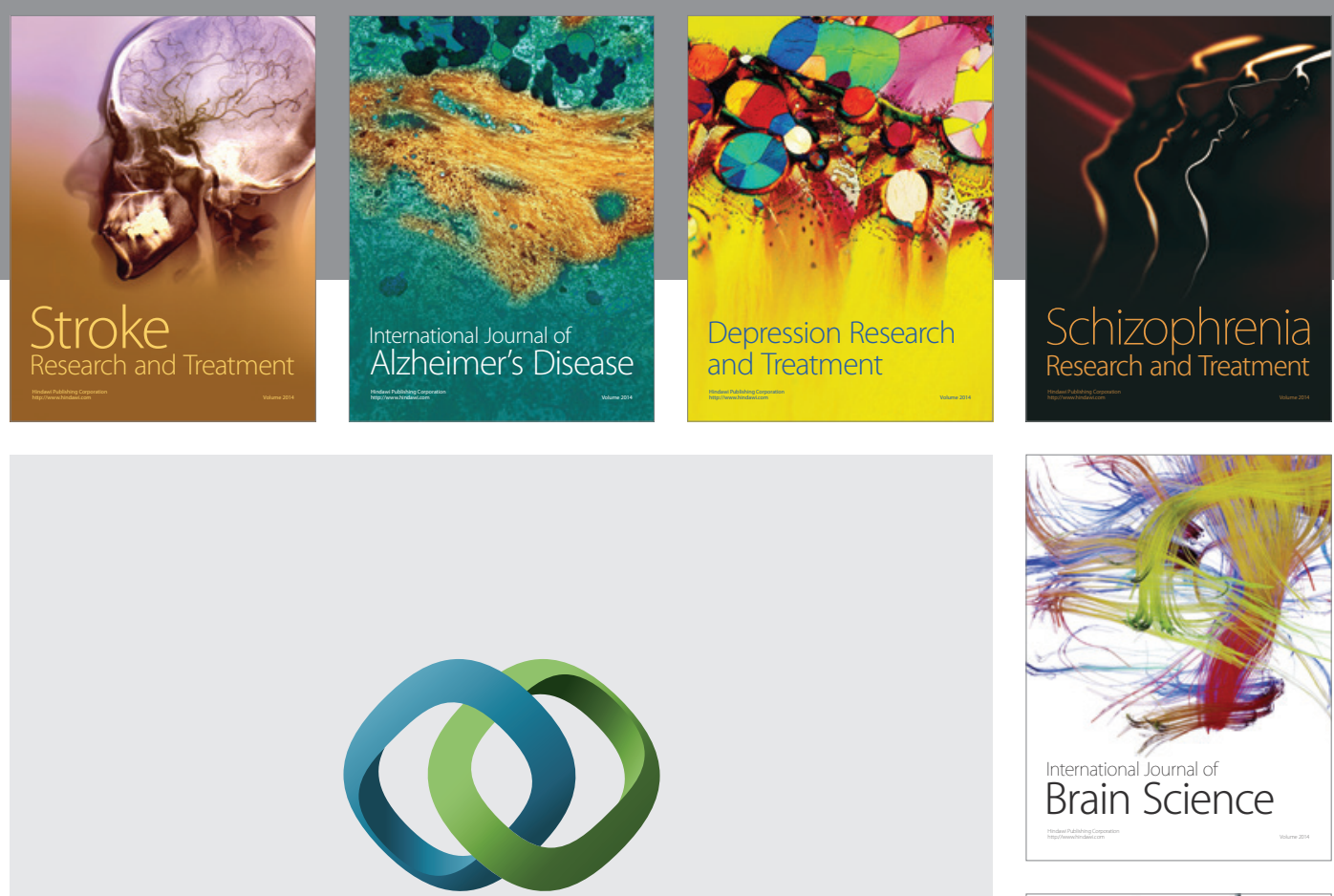

\section{Hindawi}

Submit your manuscripts at

https://www.hindawi.com
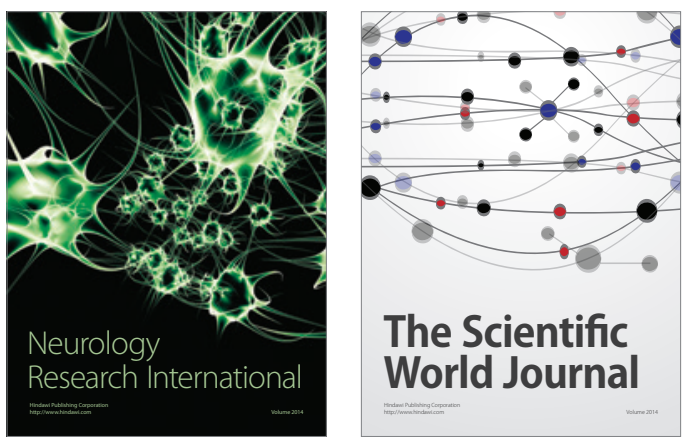

The Scientific World Journal

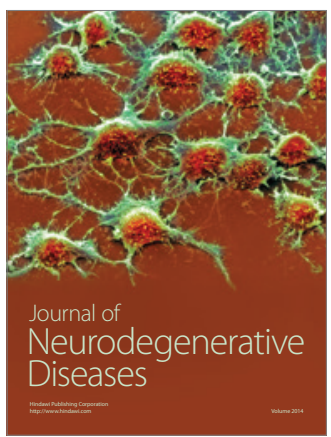

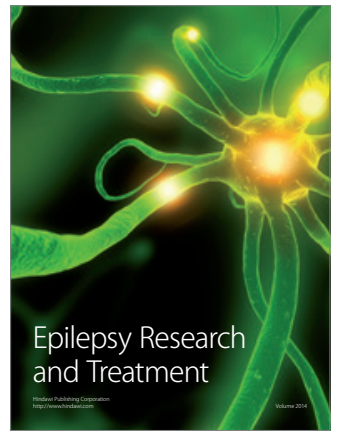

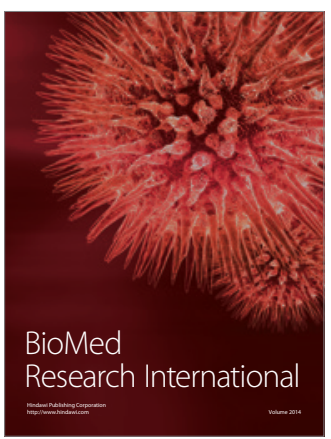

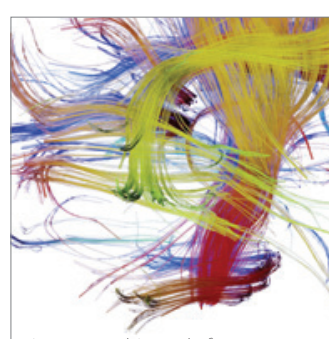

Brain Science

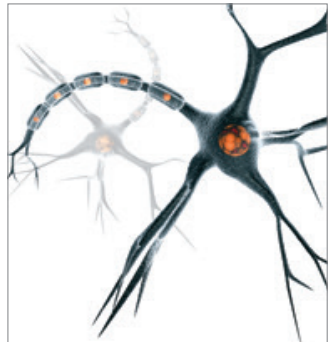

Neural Plasticity
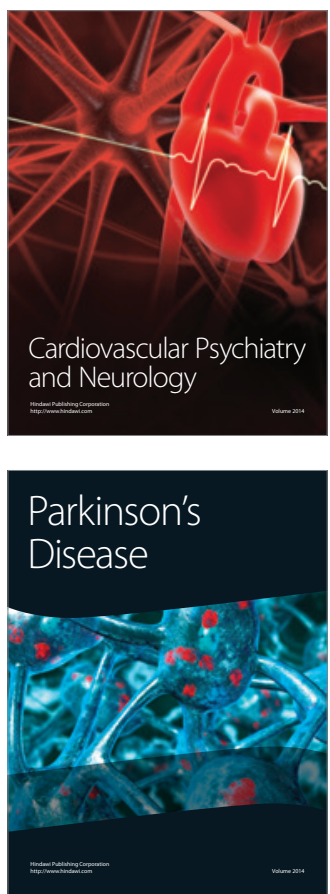